
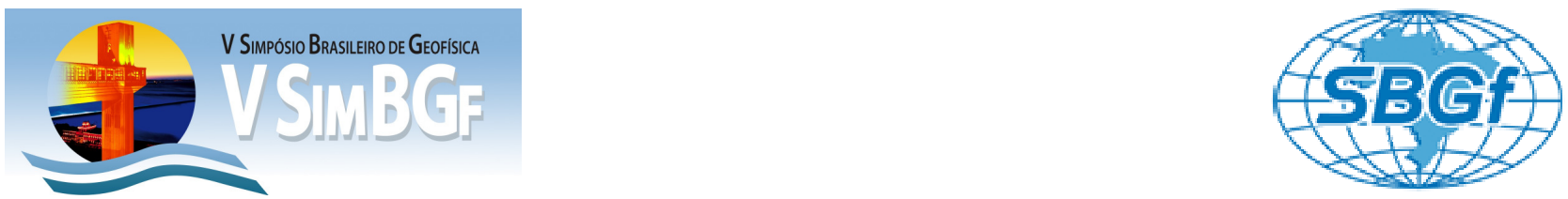

\title{
Análise dos sismos registrados na rede sismográfica local instalada na região de Santana do Acaraú, NW do Ceará, em 2009 e 2010
}

Paulo Henrique Sousa de Oliveira*1 - oliveiraphs@gmail.com

Joaquim Mendes Ferreira ${ }^{1}$, Francisco Hilário Rego Bezerra ${ }^{1}$, Aderson Farias do Nascimento ${ }^{1}$, Eduardo Alexandre Menezes de Sousa $^{1}$

1-PPGG/Universidade Federal do Rio Grande do Norte.

Copyright 2012, SBGf - Sociedade Brasileira de Geofísica

Este texto foi preparado para a apresentação no V Simpósio Brasileiro de Geofísica, Salvador, 27 a 29 de novembro de 2012. Seu conteúdo foi revisado pelo Comitê Tecnico do V SimBGf, mas não necessariamente representa a opiniáo da SBGf ou de propósitos comerciais sem prévia autorização da SBGt.

\section{RESUMO}

Em agosto de 2009, uma atividade sísmica foi registrada por estações do Laboratório Sismológico da UFRN e estava situada nas proximidades da cidade de Santana do Acaraú, localizada na região noroeste do Ceará. Em novembro do mesmo ano, foi instalada uma rede sismográfica local, possibilitando o estudo da atividade sísmica revelada e constituiu de até 6 estações sismográficas digitais. Nesse estudo, foram analisados os sismos registrados, no mínimo, em três estações, objetivando a determinação do diagrama de Wadati, de um modelo de velocidades e dos parâmetros hipocentrais. Para a determinação hipocentral, foi utilizado um modelo de semi-espaço homogêneo, com

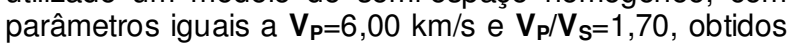
a partir da análise dos dados. Foram encontrados sismos espalhados em diversas áreas. Encontramos uma zona sísmica ativa com profundidade variando entre $3,5 \mathrm{~km}$ e $4,8 \mathrm{~km}$, com cerca $2,5 \mathrm{~km}$ de extensão na horizontal com direção NW-SE. Apesar da região possuir considerável proximidade ao Lineamento Transbrasiliano, não foi possível obter correlação entre a sismicidade e o mesmo.

\section{INTRODUÇÃO}

A cidade de Santana do Acaraú está localizada na porção noroeste do estado do Ceará, região nordeste do Brasil. Devido ao início de uma sismicidade nas proximidades dessa cidade em agosto de 2009, técnicos do Laboratório Sismológico da UFRN (LabSis) instalaram uma rede sismográfica local com 6 estações digitais (rede SA). A rede SA foi constituída por equipamentos pertencentes ao Pool de Equipamentos Geofísicos do Brasil (PEGBr), sediado no Observatório Nacional e com financiamento da PETROBRAS. Cada estação era composta por um sensor triaxial de período curto L4C3D (Sercel), um registrador DAS 130 (Reftek) e possuíam polaridade invertida. A rede SA operou entre dezembro de 2009 e dezembro de 2010 com duas configurações. A primeira configuração foi mantida entre dezembro de 2009 e setembro de 2010 e a segunda, de outubro até dezembro de 2010. Antes da instalação da rede SA, outra rede sismográfica local operou recentemente na região noroeste do Ceará, nas proximidades de Sobral e da Serra da Meruoca (rede SB). Os resultados da análise dos dados da rede SB, mostrados em Oliveira et al. (2010), contribuíram para um melhor entendimento da recente e numerosa sismicidade ocorrente na área estudada. Juntando-se a isso, a manifestação de várias áreas sísmicas e recentes eventos ocorridos na região noroeste do Ceará, temos uma das principais regiões de atividade sísmica ativa do nordeste do Brasil. Os resultados expostos nesse trabalho são provenientes das análises realizadas com os dados registrados pela rede SA.

\section{ÁREA DE ESTUDO}

A região onde se encontra a atividade sísmica de Santana do Acaraú está localizada na parte nordeste do Lineamento Transbrasiliano (LT, Figura 1). O LT é uma descontinuidade de magnitude continental, que se estende por quase $2700 \mathrm{~km}$ do nordeste até o sudeste do Brasil e atravessa a parte noroeste do Ceará, sendo designado nesse local como Lineamento Sobral - Pedro II, dividindo dois dos principais domínios geotectônicos da Província Borborema, o Domínio Noroeste do Ceará e o Domínio Ceará Central.

\section{METODOLOGIA E PROGRAMAS UTILIZADOS}

Analisamos sismos registrados, no mínimo, em três estações. Para fazer a leitura dos tempos de chegada e das polaridades das ondas $\mathrm{P}$ e $\mathrm{S}$, utilizamos o programa SAC (Tapley \& Tull, 1991). Os epicentros, hipocentros e o modelo de velocidade foram determinados com o programa HYPO71 (Lee \& Lahr, 1975). Para determinar os parâmetros do modelo de crosta utilizado, inicialmente foi necessário encontrar um conjunto confiável de dados utilizando o diagrama de Wadati (Kissingler \& Engdahl, 1973), de forma conjunta. Sobre tais parâmetros, adotamos que a velocidade da onda $P\left(\mathbf{V}_{\mathbf{P}}\right)$ e a razão $\mathbf{k}=\mathbf{V}_{\mathbf{p}} / \mathbf{V}_{\mathbf{s}}$ são consideradas constantes. As relocações hipocentrais foram realizadas com auxílio do programa HYPODD, que realiza relocações de sismos utilizando a técnica das duplas-diferenças (DD), levando vantagem do fato de que a separação hipocentral entre dois sismos é menor que a distância entre o evento e a estação e que as trajetórias dos raios percorridos entre a fonte sísmica e uma estação comum são similares ao longo de quase toda a trajetória (Waldhauser \& Ellsworth, 2000). 

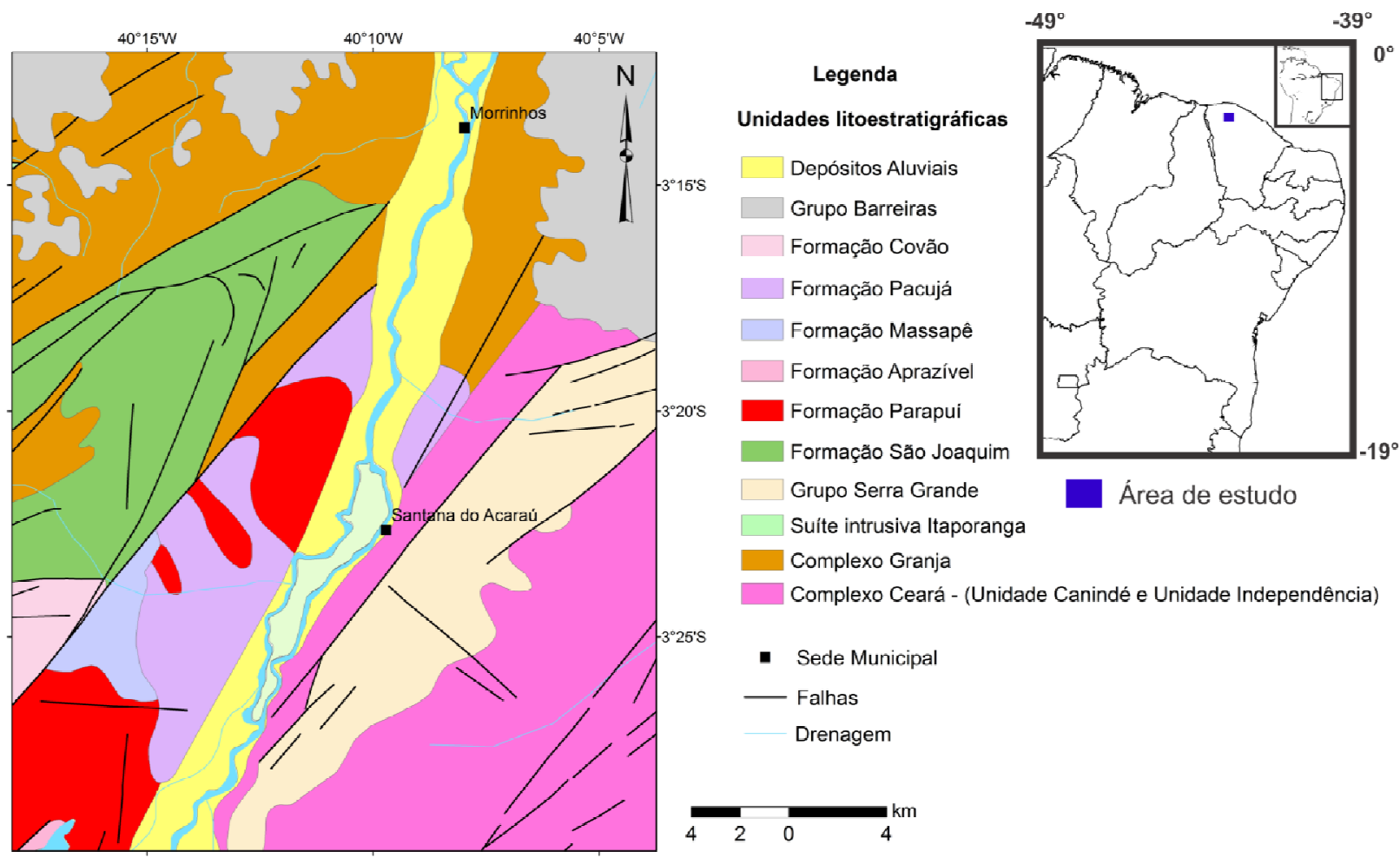

Figura 1 - Mapa geológico simplificado da área de estudo e sua localização no contexto regional.

\section{RESULTADOS E DISCUSSÃO \\ Diagrama de Wadat}

Durante a operação da rede SA, 83 sismos foram registrados em três ou mais estações e foram selecionados para obter resultados. Desses 83 sismos, 66 foram registrados, no mínimo, em quatro estações, sendo utilizados para a execução do referido diagrama. Assim, foi selecionado um conjunto confiável de 55 sismos (Figura 2), e o coeficiente k obtido foi:

$$
\mathbf{k}=\frac{\mathbf{V}_{\mathbf{p}}}{\mathbf{V}_{\mathbf{S}}}=1,701 \pm 0,0038
$$

\section{Modelo de Velocidades e Hipocentros}

O conjunto consistente de 55 sismos obtido pelo diagrama de Wadati foi utilizado na determinação do conjunto de parâmetros com maior quantidade de sismos que tiveram as menores médias de erros. Inicialmente, variando $\mathbf{V}_{\mathbf{P}}$ de $5,0 \mathrm{Km} / \mathrm{s}$ a $6,4 \mathrm{Km} / \mathrm{s}$ e $\mathrm{k}=\mathbf{V}_{\mathrm{p}} / \mathbf{V}_{\mathbf{s}}$ de 1,60 a 1,74 (Bormann, 2002) e usando o programa HYPO71, foram observados muito conjuntos de sismos com $\mathbf{V}_{\mathbf{P}} / \mathbf{V}_{\mathbf{S}}$ próximos de 1,70 , valor bem próximo do obtido com o diagrama de Wadati. Então, fixando este valor, variando $\mathbf{V}_{\mathbf{p}}$ e testando os valores nas localizações hipocentrais, obtivemos menores erros verticais, horizontais e de RMS com os seguintes parâmetros: $V_{p}=6,00 \mathrm{~km} / \mathrm{s}$ e $k=1,70$. Esse modelo foi escolhido e será utilizado em todos os cálculos futuros.

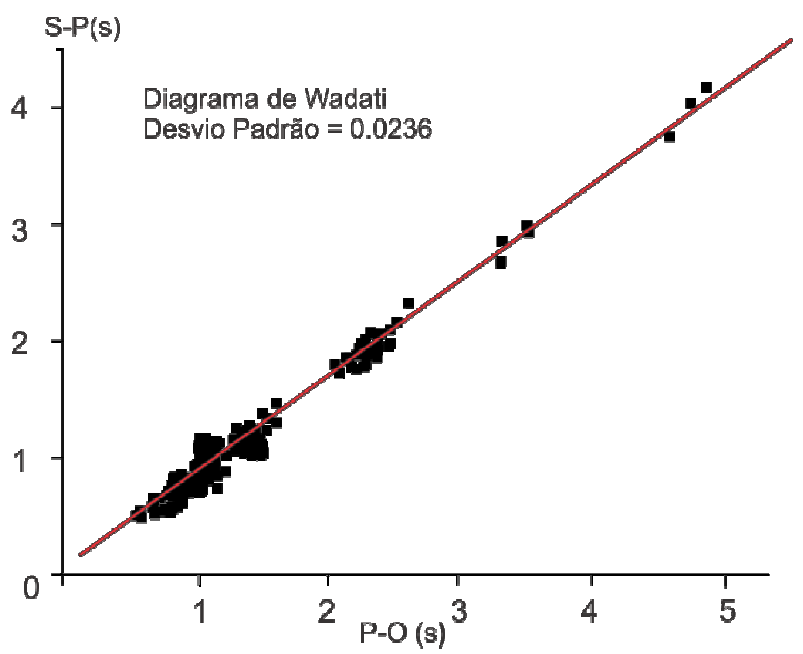

Figura 2 - Diagrama de Wadati, mostrando os sismos (quadrados pretos) constituintes do conjunto consistente de dados selecionados.

O modelo de crosta adotado foi um semi - espaço infinito, homogêneo e isotrópico, o qual fornece trajetórias de raios, provenientes das ondas $P$ e $S$, em forma de linhas retas. Esse modelo torna-se aceitável, pois a região sísmica está localizada sobre embasamento Pré Cambriano, o qual é constituído de rochas consolidadas e de baixa atenuação. Em geral, isso acarreta, nos 
sismogramas, chegadas das ondas $\mathrm{P}$ e $\mathrm{S}$ bem definidas (Figura 3).

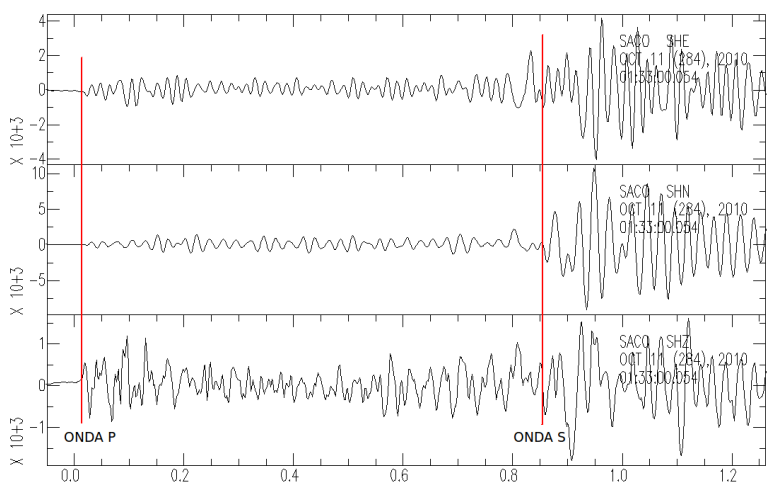

Figura 3 - Sismograma registrado na estação SACO de um evento ocorrido em 11 de outubro de 2010, às 01h33min (GMT), registrado em cinco estações da rede SA. $O$ sismograma mostra P e S bem claras. SHE - componente $E-W$, SHN - componente N-S e SHZ - componente vertical.

A variação da atividade sísmica durante o período de operação em que foram registrados dados na rede SA está mostrada na figura 4, mostrando que o seu pico, com 45 sismos, foi observado no dia 5 de março de 2010.

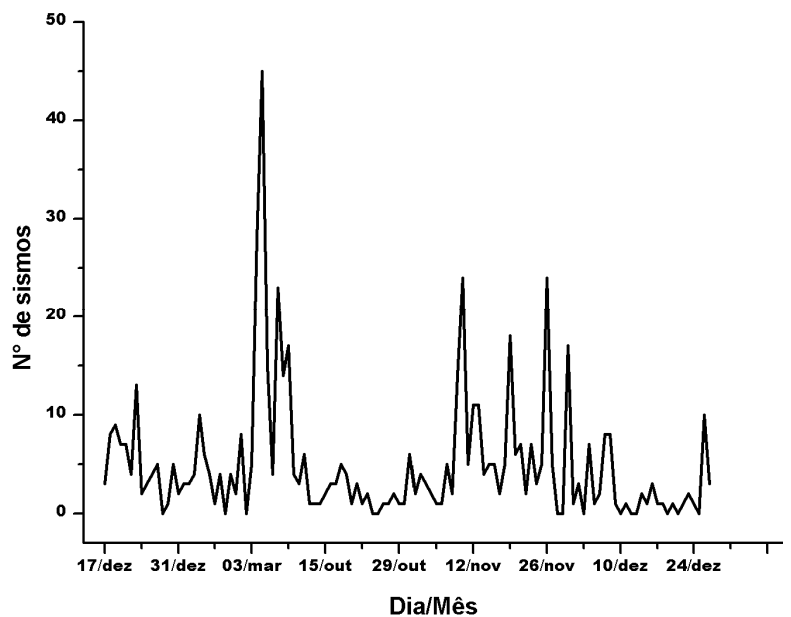

Figura 4 - Número de sismos por dia registrados durante toda a operação da rede SA. Apenas intervalos em que foram registrados dados.

\section{Primeira Configuração}

$\mathrm{Na}$ primeira configuração de rede utilizada foram registrados 38 sismos, no mínimo, em três estações. $\mathrm{Na}$ figura 5 são mostrados os epicentros calculados com auxílio do programa HYPO71.

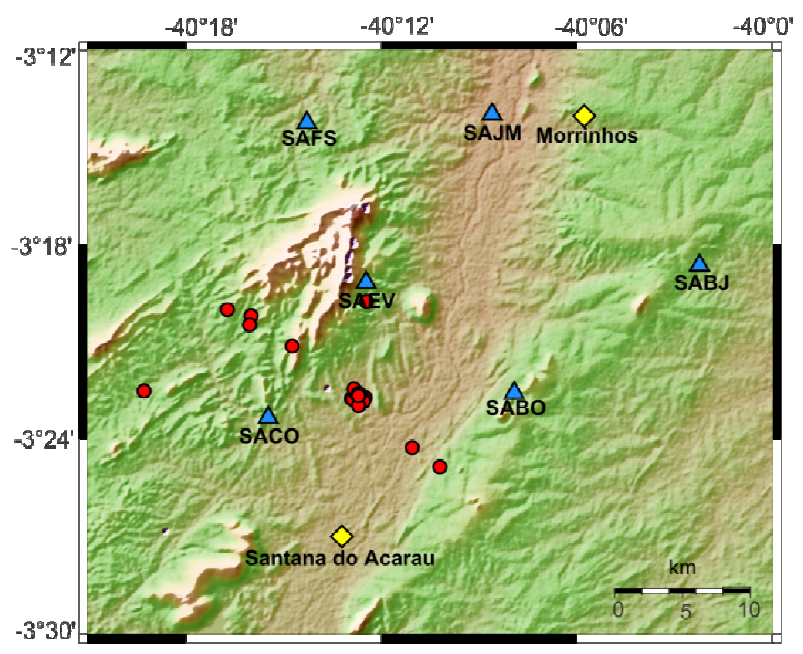

Figura 5 - Mapa epicentral dos 38 sismos registrados (círculos vermelhos), no mínimo, em três estações sismográficas, durante a primeira configuração da rede $S A$; com os seguintes critérios: $R M S \leq 0,04 \mathrm{~s} ; E R H \leq 0,35 \mathrm{~km} ; E R Z \leq 0,35 \mathrm{~km} ; N O \geq 6$.

\section{Segunda Configuração}

Nessa configuração, foram registrados 66 sismos, no mínimo, em três estações. O mapa epicentral obtido está representado pela figura 6 .

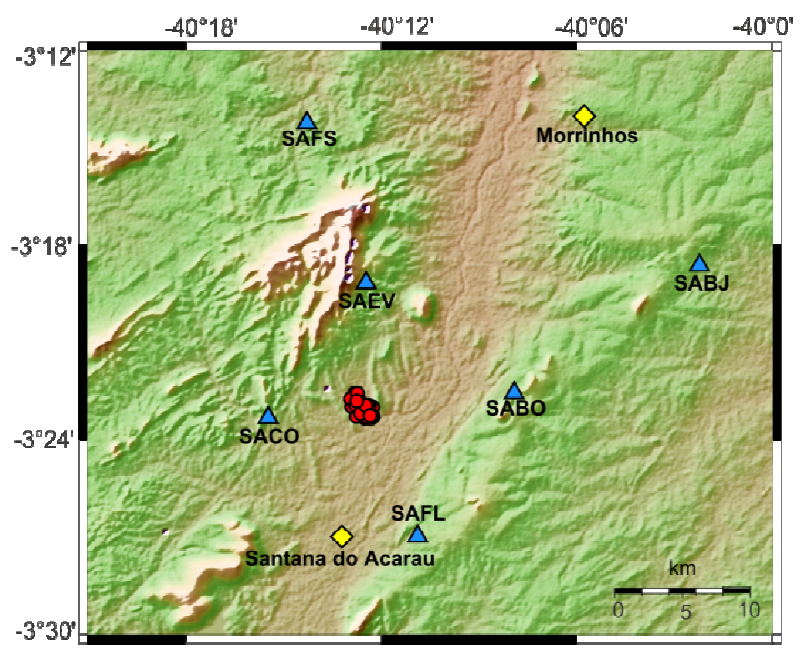

Figura 6 - Mapa epicentral dos 66 sismos registrados (círculos vermelhos), no mínimo, em três estações sismográficas, durante a segunda configuração da rede $S A$, utilizando os seguintes critérios: $R M S \leq 0,04 \mathrm{~s} ; E R H \leq 0,35 \mathrm{~km} ; E R Z \leq 0,35 \mathrm{~km} ; N O \geq 6$.

$\mathrm{Na}$ figura 5 os sismos tendem a se espalhar na direção NW-SE, apesar do aglomerado principal. O aglomerado mostrado na figura 6, não mostrou alinhamento preferencial em nenhuma direção. Nessa segunda configuração de rede, os erros verticais, horizontais e de RMS foram menores, comparado com aqueles calculados com os dados obtidos com a primeira configuração de rede. 


\section{Relocação hipocentral}

Utilizamos dados de catálogo com fases das ondas P e S para executarmos o programa HYPODD. Durante a execução, foram testados vários parâmetros e obtemos sismos ligados e bem conectados. Após várias execuções com os seguintes parâmetros: MAXSEP $=5 \mathrm{~km}$, MAXDIST $=100 \mathrm{~km}$, foram relocados 56 sismos bem conectados, como mostrados na figura 7 . Os sismos relocados possuem certo alinhamento preferencial quase que igual ao alinhamento observado na figura 5 , a qual contém os sismos analisados na primeira configuração da rede SA.

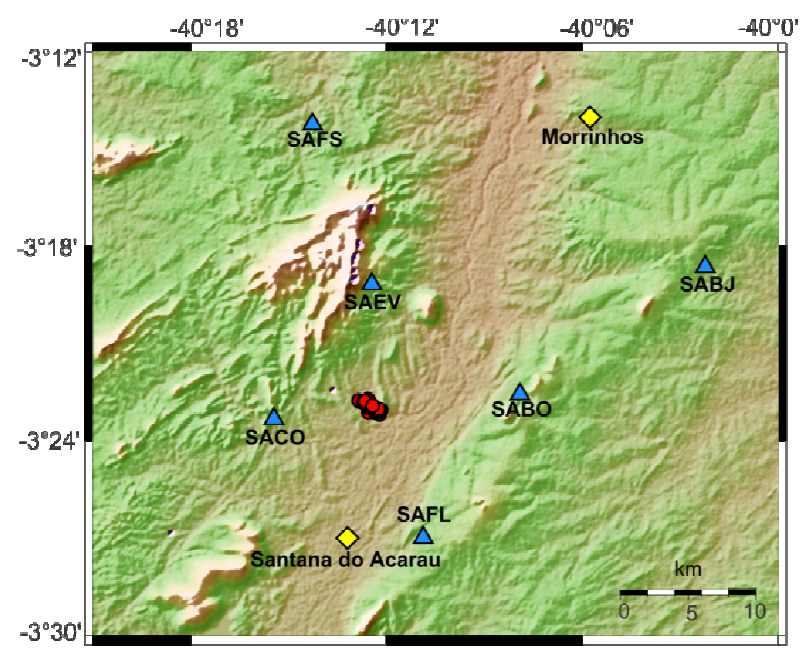

Figura 7 - Mapa de relocação hipocentral utilizando os sismos registrados, no mínimo, em 4 estações da rede $S A$

\section{CONCLUSÔES}

A sismicidade analisada nesse estudo encontra-se espalhada em várias áreas nas proximidades das cidades de Santana do Acaraú e Morrinhos, mas somente os principais sismos foram mostrados nos mapas elaborados. A análise detalhada da distribuição hipocentral e das polaridades das ondas $P$ dos sismos locais registrados na rede $S A$, evidenciam que há uma falha sismogênica na direção NW-SE. A sismicidade em ênfase aqui está bem próxima ao LT, mas em uma direção quase perpendicular ao mesmo. No entanto, mais uma vez, não foi possível correlacionar a sismicidade com uma possível reativação, mostrando mais uma vez, que nem sempre é possível obter correlação entre sismicidade e falhas mapeadas. Atualmente, na área de estudo, não há evidência que possa concluir que o LT está reativado.

\section{AGRADECIMENTOS}

Os Autores agradecem aos técnicos do LabSls pela instalação, operação e manutenção da rede de estações localizada na região de Santana do Acaraú. Ao Dr. Marcelo Assumpção, do IAG/USP, pelo auxílio na análise dos dados. Ao INCT-ET pelos recursos financeiros e ao $\mathrm{PEGBr}$ pelos equipamentos de última geração utilizados. PHSO agradece à CAPES pela bolsa de doutorado.

\section{REFERÊNCIAS}

BEZERRA, F.H.R., do NASCIMENTO, A.F., FERREIRA, J.M., NOGUEIRA, F.C., FUCK, R.A., BRITO NEVES, B.B., SOUZA, M.O.L. 2011. Review of active faults in the Borborema Province, Intraplate South America Integration of seismological and paleoseismological data, Tectonophysics.

BORMANN, P., BAUMBACH, M., BOCK, G., GROSSER, H., CHOY, G.L AND BOATWRIGHT. 2002. IASPEI - New Manual of Seismological Observatory Practice. Vol 1.

FERREIRA, J.M., OLIVEIRA, R.T., TAKEYA, M.K. \& ASSUMPÇÃO, M. 1998. Superposition of local and regional stress in northeast Brazil: evidence from focal mechanism around the Potiguar marginal basin. Geophys. J. Int., 134: 341-355.

KISSSLINGER, C. \& ENGDAHL, E.R. 1973. The interpretation of the Wadati diagram with relaxed assumptions. Bull.Seism.Soc.Am. 63: 1723-1736.

LEE, W.H.K. \& LAHR, J.C. 1975. HYPO71 (revised): a computer program for determining hypocenter, magnitude and first motion pattern of local earthquakes. U.S. Geol. Surv. Open File Rep. 75-311, 114 pp.

LEE, W.H.K, STEWART, S.W. 1981. Principles and Aplications of Microearthquake Networks. Academic Press, Inc (London), LTD.

OLIVEIRA, P.H.S., FERREIRA, J.M., do NASCIMENTO, A.F., BEZERRA, F.H.R., SOARES, J.E., FUCK, R.A., 2010. Estudo da Sismicidade na Região de Sobral - CE, NE do Brasil, em 2008. IV Simpósio da Sociedade Brasileira de Geofísica. Brasília - DF.

SANTOS, T.J.S., FETTER, A.H, HACKSPACHER, P.C., NOGUEIRA NETO, J.A. 2008. Neoproterozoic Tectonic and Magmatic Episodes in the NW Sector of Borborema Province, NE Brazil, During Assembly of Western Gondwana. Journal of South American Earth Sciences. 25: 271-284.

SYKES, L.R. 1978. Intraplate seismicity, reactivation of preexisting zones of weakness alkaline mamatism, and other tectonism posdating continental fragmentation. Rev. Geophys. Space Phys., 16: 621-688.

TAPLEY W.C. \& TULL. J.E. 1991. SAC-Seismic Analysis Code, USER MANUAL. Livermore National Laboratory, $413 \mathrm{pp}$.

WALDHAUSER, F., ELLSWORTH, W.L. 2000. A Double Difference Earthquakes Location Algorithm: Method and Application to the Northern Hayward Fault, California. Bulletim of the Seismological Society of America. v.90, n.6, pp.1353-1368. 